Research Paper

\title{
Identification and Validation of a Five MicroRNA Sig- nature Predictive of Prostate Cancer Recurrence and Metastasis: A Cohort Study
}

\author{
Robert K. Nam ${ }^{\circledR}$, Yutaka Amemiya ${ }^{2}$, Tania Benatar², Christopher J.D. Wallis ${ }^{1}$, Jessica Stojcic-Bendavid ${ }^{1}$, \\ Stephanie Bacopulos², Christopher Sherman³, Linda Sugar³, Magda Naeim³, Wenyi Yang2, Aiguo Zhang2, \\ Laurence H. Klotz ${ }^{1}$, Steven A. Narod ${ }^{4}$, and Arun Seth ${ }^{2}$ \\ 1. Division of Urology, Sunnybrook Research Institute, Sunnybrook Health Sciences Centre, University of Toronto, 2075 Bayview Ave., Toronto, ON, \\ M4N3M5, Canada. \\ 2. Department of Anatomic Pathology, Platform Biological Sciences, Sunnybrook Research Institute, Sunnybrook Health Sciences Centre, University of To- \\ ronto, 2075 Bayview Ave., Toronto, ON, M4N3M5, Canada. \\ 3. Department of Laboratory Medicine and Pathobiology, Sunnybrook Research Institute, Sunnybrook Health Sciences Centre, University of Toronto, 2075 \\ Bayview Ave., Toronto, ON, M4N3M5, Canada. \\ 4. Department of Public Health Sciences, University of Toronto, 790 Bay St., Toronto, ON, M5G 1N8, Canada.
}

$\triangle$ Corresponding authors: Robert K. Nam, M.D. or Arun Seth, Ph.D., Sunnybrook Health Sciences Centre, Division of Urology, 2075 Bayview Ave., MG-406, Toronto, Ontario, Canada, M4N 3M5. Phone: (416) 480-5075; FAX: (416) 480-6121; E-mail: robert.nam@utoronto.ca; E-mail: arun.seth@utoronto.ca

( 2015 Ivyspring International Publisher. Reproduction is permitted for personal, noncommercial use, provided that the article is in whole, unmodified, and properly cited. See http://ivyspring.com/terms for terms and conditions.

Received: 2015.07.30; Accepted: 2015.08.02; Published: 2015.09.15

\begin{abstract}
Background: MicroRNA (miRNA) have been shown to be important in regulating gene expression in prostate cancer. We used next generation miRNA sequencing to conduct a whole miRNome analysis to identify miRNAs associated with prostate cancer metastasis.

Methods: We conducted discovery and validation analyses of miRNAs among a total of 546 men who underwent surgery for prostate cancer using the development of metastasis as an endpoint. Genome wide analysis was conducted among the discovery group $(n=31)$ to identify new miRNAs associated with prostate cancer metastasis. Selected miRNAs were then analyzed using qPCR on prostatectomy specimens from an independent cohort $(n=515)$ to determine whether their expression could predict the development of metastasis after surgery. To examine the biology underlying these associations, we created prostate cancer cell lines which overexpressed miR-30la for in vitro and in vivo functional assays.

Results: We identified 33 miRNAs associated with prostate cancer metastasis and selected a panel comprising miRs-30la, 652, 454, 223 and 139 which strongly predicted metastasis (AUC=95.3\%, 95\%C.l.:84\%-99\%). Among the validation cohort, the 15-year metastasis-free survival was $77.5 \%$ (95\% C.I.:63.9\%-86.4\%) for patients with a high miRNA panel score and $98.8 \%$ (95\% C.I.:94.9\%-99.7\%, $p<0.0001$ for difference) for those with a low score. After adjusting for grade, stage, and PSA, the hazard ratio for metastasis was $4.3(95 \%$ C.I.: 1.7-11.1, $\mathrm{p}=0.002)$ for patients with a high miRNA panel score, compared to those with a low score. Prostate cancer cell lines overexpressing miR-30la had in significantly higher tumor growth and metastasis in a xenograft mouse model.

Conclusions: A panel of miRNAs is associated with prostate cancer metastasis. These could be used as potential new prognostic factors in the surgical management of prostate cancer.
\end{abstract}

Key words: MicroRNA; Prostatic neoplasms; Prostatectomy; High-throughput nucleotide sequencing 


\section{Introduction}

One of the most important issues in managing patients with localized prostate cancer is the selection of appropriate treatment. A significant proportion of patients with prostate cancer have indolent disease, whereas others have highly aggressive cancers that will metastasize. It would be helpful to identify biologic markers that allow us to distinguish between the two forms so that appropriate treatment can be offered. All current treatments are associated with side effects that may compromise quality of life and, when possible, men wish to avoid unnecessary treatment (1). Currently, established prognostic factors for prostate cancer include grade, stage and serum prostate specific antigen level (PSA) at diagnosis (2). However, these cannot unambiguously stratify patients between those with indolent and aggressive forms of prostate cancer.

It has been established that microRNAs (miRNA) are key regulators of gene expression in cancer (3). MiRNAs are small, noncoding RNAs that regulate gene expression at the post-transcriptional and translational levels and have been found to modulate differentiation, proliferation and apoptosis (4). Prior to initiating this study, no others had published miRNA profiles of human prostate cancers using a genome-wide approach or correlated the prognostic significance of observed variation in miRNA expression in prostate cancer. Recently published work has shown that numerous different miRNAs may be prognostically important in prostate cancer progression (5-8). In this study, we sought to identify miRNA-based biomarkers that correlate with the presence of metastatic disease and then to test whether or not these miRNAs predict metastasis after surgery for clinically localized prostate cancer.

\section{Materials and Methods}

This study was approved by the Sunnybrook Health Sciences Centre Research Ethics Board and written, informed consent was obtained from all patients.

\section{MicroRNA Gene Discovery using Whole miRNome Analysis}

To identify candidate miRNAs associated with aggressive prostate cancer, we selected a Discovery Set of patients with and without metastatic disease from an established University of Toronto tumour bank (9). Cases were men who recurred a minimum of five years after surgery and who developed metastatic disease $(n=18)$. Controls were cancer-free at least five years post-surgery $(n=13)$. Cases and controls were matched for grade, stage, PSA level and year of surgery. We compared the expression levels of miRNAs from their tumour tissue across the whole miRNome using next-generation miRNA sequencing methods. For each subject, formaldehyde-fixed paraffin-embedded (FFPE) tissue of the radical prostatectomy specimen was identified. All slides were re-reviewed and a representative slide was selected for each of the specimens, marking the areas of cancer. The corresponding tumour block from where the slide originated was then used. In each case the paraffin block chosen for analysis was the one with the largest focus of tumor seen on the corresponding histologic slide, and tissue was extracted (cored) from the area of the block with the highest tumor-to-stroma ratio as identified on the slide. Two FFPE cores with a diameter of $1 \mathrm{~mm}$ and a maximum length of $3 \mathrm{~mm}$ after trimming of excess paraffin were used for total RNA extraction.

\section{Whole miRNome Analysis}

Whole miRNome analysis for miRNA expression between cases and controls was performed using the Life Technologies SOLiD5500xl next generation sequencer. Total RNA from FFPE cores was extracted by the Recoverall Total Nucleic Acid Isolation Kit for FFPE (Life Technologies). Small RNA fraction was enriched using the Purelink miRNA Isolation kit (Life Technologies). All procedures were carried out in accordance with the manufacturer's manuals.

cDNA libraries for sequencing were constructed using the SOLiD Total RNA-Seq Kit (Life Technologies). For microRNA-seq, SOLiD adaptors were ligated to small RNA followed by reverse transcription to generate a cDNA library. cDNA products between $60 \mathrm{bp}$ and 70bp were purified from 10\% TBE-urea gel followed by PCR amplification with barcode primer using RNA barcoding Kit (Life Technologies). The bar-coded cDNA libraries were pooled and drove onto beads to generate bead clones by emulsion PCR. Each 3' modified beads were deposited onto a Flowchip. 35bp single end sequence reads for microRNA-seq were generated using SOLiD 5500xl next generation sequencer.

The sequencing data were analyzed by Geospiza's Genesifter analysis edition version v4.0. Preset data analysis pipeline, small RNA (v2) for microRNA-seq were used. SOLiD adapters, mitochondrial RNA, ribosomal RNA and small nuclear RNA were filtered out. The remaining sequencing data were mapped onto Human reference sequence, NCBI Build 36 for microRNA.

For primary data comparison, a panel of the top five miRNAs (up- or down-regulated) associated with prostate cancer metastasis were selected based on the magnitude of the fold-change in expression (minimum of 1.75) and the strength of association (adjusted p-value $<0.05$ ) by using the Genesifter software. 


\section{Cohort Validation}

To determine whether the five-miRNA panel could be used as an independent prognostic marker for patients with localized prostate cancer, we examined a cohort of patients who underwent surgery for prostate cancer who were separate and independent from the initial Discovery Set. Study subjects were selected from a cohort of 560 patients who underwent radical prostatectomy for clinically-localized prostate cancer between 1990 to 2000 at a single, tertiary care centre (Sunnybrook Health Sciences Centre). Patients were excluded if they had a history of other non-melanoma cancer $(n=7)$, incomplete medical chart information $(n=15)$, incomplete pathology tumour information $(n=9)$, or had neoadjuvant hormone therapy $(n=14)$, leaving 515 patients for analysis. The primary endpoint was the development of bone or visceral metastasis, based on medical imaging evaluation. The secondary endpoint was the development of biochemical recurrence based on serum prostate specific antigen (PSA) levels.

Each of the five microRNAs was measured using quantitative PCR techniques from paraffin-embedded tumour blocks. Each prostatectomy specimen was re-reviewed and the most dominant pattern based on the Gleason Score was chosen for tumour extraction. PCR reactions for each sample were carried out in triplicate. Each miRNA expression level was normalized with most stably expressed miRNA through all patient groups in our deep-sequencing data (miR-28).

For the validation cohort, the medical records were systematically reviewed using standardized data entry forms by trained data abstractors and stored within a prostate cancer-specific database. Clinical follow-up consisted of four assessments in the year following surgery, two assessments in the second year and one assessment every year thereafter. At each follow-up, patients had a clinical evaluation, and a PSA test.

Biochemical recurrence was defined as a PSA increase of at least $0.2 \mathrm{ng} / \mathrm{mL}$ on at least two separate consecutive measurements that are at least 3 months apart. Metastasis was defined as lesions within the bone identified on radionuclide bone scan and lymphadenopathy or visceral lesions identified by computed tomography imaging of the abdomen, pelvis and chest. These examinations were undertaken at the discretion of the treating urologist. We compared baseline characteristics of the study population with univariate statistics: student's t-test for continuous data and chi-squared test for categorical variables. Patients were considered to be at risk from the date of surgery until recurrence or until the date of the last PSA test. Patients that were lost to follow-up were censored at the date of their last PSA test or follow-up.
Multivariate Cox proportional hazard modeling was used to evaluate the prognostic significance of the microRNA panel.

A risk score for each patient was developed by linear combination of the expression level of each miRNA, weighted by the regression coefficients derived from the univariate logistic regression model based on the case-control design(10, 11). The following formula was used to derive the risk score for the Discovery Set:

RNA Risk Score $=1.83 \times\{$ miR-652 $\}+2.84 \times\{$ miR-301a $\}$ $+1.68 \times\{$ miR-454 $\}-1.50\{$ miR-139\} $-2.85 \times\{$ miR-223 $\}$

For the Validation Set, we used univariate Cox-proportional hazard models based on the cohort study design to develop the linear formula:

RNA Risk Score $=2.42 \times\{$ miR-652 $\}+8.89 \times\{$ miR-301a $\}$

$+13.82 \times\{$ miR-454\} $-1.10 \times\{$ miR-139 $\}-0.99 \times$

$\{\mathrm{miR}-223\}$

\section{Animals}

A colony of immunodeficient NOD.CB17 Prkdscid /J mice (Jackson Labs, stock number 001303 (termed NOD/SCID), mice were maintained in-house under aseptic sterile conditions. Mice were given autoclaved food and water. All procedures were approved by the Institutional Research Ethics Board and the Animal Care Committee. Mice were sacrificed upon sign of heavy tumor burden, signs of severe respiratory distress, or weakness and lethargy.

\section{Plasmid constructs and establishment of stable transfectants}

pCMV-MIR plasmid containing human miR-301a (MI0000745, Origene) or empty vector control (pCMVMIR, Origene) was transfected into PC3 or LNCAP cells using Lipofectamine ${ }^{\mathrm{TM}} 2000$ transfection reagent (Life technologies). Briefly, for $10 \mathrm{~cm}^{2}$ surface area, $4 \mu \mathrm{g}$ of plasmid DNA was transfected together with $10 \mu \mathrm{l}$ of Lipofectamine ${ }^{\mathrm{TM}}$ 2000. Medium was changed 4-6 hours post-transfection. Cells were passaged into fresh media 24 hours post-transfection. Selection reagent, G418 $(400 \mu \mathrm{g} / \mathrm{ml})$, was added 48 hours post-transfection. Once stable cells were established, the GFP+ population was further selected by flow cytometric cell sorting. From cell-sorted populations, subclones were generated by expanding GFP+ cells by plating 0.9 cells/well.

\section{Proliferation Assay - Thymidine Assay}

In order to assay growth, stably transfected prostate cancer cells were seeded at 103 cells/well into flat-bottomed 96-well plates ( $\mathrm{n}=3-6$ wells) in DMEM high glucose media containing $0.5 \%$ FBS for miRNAs 301a, 652 and 454, or 5\% FBS for miRNAs 139 and 223. Cells were harvested from wells at vari- 
ous time points following a $6-\mathrm{h}$ incubation with 1 $\mu \mathrm{Ci} / \mathrm{ml}[3 \mathrm{H}]$ thymidine, and scintillation was quantified by TopCount Microplate Scintillation Counter.

\section{Reverse transcription and Quantitative Real time PCR}

Total RNA was extracted using the Recoverall Total Nucleic Acid Isolation Kit (Life Technologies) using miRNeasy Mini Kit (Qiagen) from cell lines according to the manufacturers' instructions. One hundred nanogram of total RNA was reverse-transcribed using the miScript II RT kit (Qiagen) for miRNA using the QuantiTect Reverse Transcription Kit (Qiagen) for mRNA. Quantitative real-time PCR was performed in triplicate by using miScript SYBR Green PCR kit (Qiagen) for miRNA on StepOnePlus Real Time PCR system (Life Technologies) with specific primer pairs. The primers for quantification of mature microRNA were purchased from Qiagen. Primers are miR-28 (endogenous control; MS00009254), miR-301a (MS00009317), miR-652 (MS00010451), miR-454 (MS00007861), miR-223 (MS00003871) and miR-139 (MS00003493).

\section{Xenograft tumorigenicity}

Empty vector or miR-301a stably transfected PC3 $\left(1 \times 10^{6} /\right.$ mouse $)$ or LNCAP prostate cancer cells $(2.5 \times$ $10^{6} /$ mouse) were subcutaneously injected together with matrigel (1:1 ratio) into the fat pad of NOD/SCID mice, four animals for control cell lines and 14 for miR-301a transfected prostate cancer lines. Tumors were measured every 3-4 days. After 4 weeks, some mice were sacrificed and tumors were removed for analysis. Another set of mice had tumors removed surgically under anaesthetic, and were then followed for 6 more weeks, after which they were sacrificed, and organs (Lung and liver) were harvested and examined for the presence of metastasis after fixing and H\&E staining of tissue sections. Quantitative imaging scores for anti-human-low molecular weight keratin in lungs of mice xenografted with miR-301 transfected LNCaP and PC3 cells were obtained by using Leica SCN400.

\section{Results}

The discovery set comprised 31 patients treated with radical prostatectomy for prostate cancer. Baseline demographic and tumor characteristics of the cases and controls are described in Table 1. From the Discovery Set, a total of 33 miRNAs were identified using pre-defined selection criteria. Of the $33 \mathrm{miR}-$ NAs, 29 were up-regulated and 4 were down-regulated (Table 2). Five miRNAs (onco-miRs 301a, 652, 454, and tumour suppressive-miRs 139 and 223) were selected for further investigation. Among the up-regulated miRNAs, miR-301a and miR-652, had expression levels greater than 2.0 fold between cases and controls, and the lowest adjusted p-values for association. The miRNA with the lowest $p$-value for association, miR-671 had a low ratio of expression and was not selected. MiR-545, miR-205, miR-1302, mir-501, mir-1249 and mir-154 had very low absolute expression levels indicating a high likelihood of poor reproducibility during $\mathrm{qPCR}$ validation, and therefore, not chosen. MiR-454 is likely to co-regulate with miR-301a as they share high sequence identity and are located at the same intronic region of SKA2 (12). MiR-223 and miR-139 were the most significantly downregulated between cases and controls. This was confirmed for miR-139 and miR-223 by literature searches (13-16).

Table 1: Baseline characteristics of study cohort

\begin{tabular}{|c|c|c|c|c|c|c|}
\hline & \multicolumn{3}{|c|}{ Discovery set } & \multicolumn{3}{|c|}{ Validation set } \\
\hline & Case & Control & p-value & No recurrence & Recurrence / metastasis & $\mathrm{p}$-value \\
\hline Age (mean, SD) & $66.4(6.7)$ & $63.6(7.1)$ & 0.2650 & $61.4(7.0)$ & $63.2(6.7)$ & 0.0061 \\
\hline PSA $(\mathrm{ng} / \mathrm{mL})$ & & & $0.9481^{*}$ & & & 0.0003 \\
\hline$\leq 4$ & $2(15 \%)$ & $2(11 \%)$ & & $89(27 \%)$ & $21(13 \%)$ & \\
\hline $4-10$ & $6(46 \%)$ & $7(39 \%)$ & & $170(52 \%)$ & $95(57 \%)$ & \\
\hline$>10$ & $4(31 \%)$ & $7(39 \%)$ & & $65(20 \%)$ & $51(31 \%)$ & \\
\hline Missing & $1(8 \%)$ & $2(11 \%)$ & & N/A & N/A & \\
\hline Gleason score & & & $0.6609^{*}$ & & & $<0.0001$ \\
\hline 6 & $2(15 \%)$ & $1(6 \%)$ & & $108(33 \%)$ & $10(6.0 \%)$ & \\
\hline 7 & $6(46 \%)$ & $7(39 \%)$ & & $201(62 \%)$ & $128(77 \%)$ & \\
\hline $8-10$ & $5(38 \%)$ & $10(56 \%)$ & & $15(4.6 \%)$ & $29(17 \%)$ & \\
\hline Pathologic Stage & & & $0.0008^{*}$ & & & $<0.0001$ \\
\hline pT2 & $11(85 \%)$ & $3(17 \%)$ & & $245(76 \%)$ & $58(35 \%)$ & \\
\hline pT3a & $0(0 \%)$ & $3(17 \%)$ & & $66(20 \%)$ & $68(41 \%)$ & \\
\hline pT3b & $2(15 \%)$ & $12(67 \%)$ & & $13(4 \%)$ & $41(25 \%)$ & \\
\hline Margin positivity & $4(31 \%)$ & $11(61 \%)$ & $0.1489^{*}$ & $103(32 \%)$ & $98(59 \%)$ & $<0.0001$ \\
\hline Lymph node status & & & $0.1737^{*}$ & & & $<0.0001$ \\
\hline Negative & $9(69 \%)$ & $13(72 \%)$ & & $88(27 \%)$ & $60(36 \%)$ & \\
\hline Positive & $0(0 \%)$ & $3(17 \%)$ & & $2(0.62 \%)$ & $10(6.0 \%)$ & \\
\hline Missing & $4(31 \%)$ & $2(11 \%)$ & & $234(72 \%)$ & $97(58 \%)$ & \\
\hline
\end{tabular}

* Fisher's exact test used due to small expected cell counts. 
Table 2: List of 33 microRNAs identified by whole miRNome analysis to be associated with prostate cancer recurrence.

\begin{tabular}{|c|c|c|c|c|c|c|}
\hline \multirow[t]{2}{*}{ Gene Identifier } & \multicolumn{2}{|c|}{ Mean Expression Level ${ }^{*}$} & \multirow[t]{2}{*}{ Ratio } & \multirow[t]{2}{*}{ Direction } & \multirow[t]{2}{*}{ p-value } & \multirow[t]{2}{*}{ adj. p-valueł } \\
\hline & PNMNR & PR & & & & \\
\hline hsa-mir-671 & 6.94 & 7.75 & 1.76 & Up & $3.75 \mathrm{E}-06$ & 0.00072 \\
\hline hsa-mir-301a & 7.55 & 8.78 & 2.34 & $\mathrm{Up}$ & 2.64E-05 & 0.00124 \\
\hline hsa-mir-652 & 6.73 & 8.30 & 2.97 & Up & 3.69E-05 & 0.00124 \\
\hline hsa-mir-223 & 11.90 & 10.66 & 2.36 & Down & $7.46 \mathrm{E}-05$ & 0.00179 \\
\hline hsa-mir-545 & 4.04 & 5.10 & 2.08 & Up & 0.00049 & 0.00524 \\
\hline hsa-mir-501 & 4.91 & 5.74 & 1.77 & Up & 0.00052 & 0.00525 \\
\hline hsa-mir-1249 & 4.47 & 5.37 & 1.86 & $\mathrm{Up}$ & 0.00079 & 0.00622 \\
\hline hsa-mir-454 & 7.26 & 8.22 & 1.94 & $\mathrm{Up}$ & 0.00148 & 0.00981 \\
\hline hsa-mir-154 & 4.26 & 5.28 & 2.03 & Up & 0.00159 & 0.01016 \\
\hline hsa-mir-497 & 10.28 & 11.19 & 1.89 & Up & 0.00179 & 0.01111 \\
\hline hsa-mir-708 & 5.45 & 6.67 & 2.32 & $\mathrm{Up}$ & 0.00214 & 0.01206 \\
\hline hsa-mir-452 & 5.15 & 6.02 & 1.84 & Up & 0.00303 & 0.01385 \\
\hline hsa-mir-1302-3 & 2.75 & 4.42 & 3.18 & Up & 0.00354 & 0.01529 \\
\hline hsa-mir-1302-1 & 2.75 & 4.53 & 3.45 & Up & 0.00348 & 0.01529 \\
\hline hsa-mir-18a & 6.27 & 7.64 & 2.57 & $\mathrm{Up}$ & 0.00421 & 0.01605 \\
\hline hsa-mir-188 & 7.50 & 8.48 & 1.97 & $\mathrm{Up}$ & 0.00577 & 0.01878 \\
\hline hsa-mir-1248 & 7.77 & 8.91 & 2.21 & Up & 0.00610 & 0.01907 \\
\hline hsa-mir-9-3 & 4.96 & 6.12 & 2.23 & Up & 0.00614 & 0.01907 \\
\hline hsa-mir-1302-7 & 2.55 & 4.19 & 3.1 & Up & 0.00757 & 0.02113 \\
\hline hsa-mir-301b & 3.65 & 4.61 & 1.95 & $\mathrm{Up}$ & 0.00771 & 0.02116 \\
\hline hsa-mir-489 & 2.67 & 3.66 & 1.98 & Up & 0.00830 & 0.02183 \\
\hline hsa-mir-411 & 2.73 & 3.83 & 2.14 & Up & 0.01170 & 0.02673 \\
\hline hsa-mir-34c & 4.52 & 5.55 & 2.04 & Up & 0.01239 & 0.02800 \\
\hline hsa-mir-139 & 8.08 & 7.24 & 1.79 & Down & 0.01442 & 0.03061 \\
\hline hsa-mir-433 & 3.15 & 4.21 & 2.09 & $\mathrm{Up}$ & 0.01400 & 0.03061 \\
\hline hsa-mir-624 & 4.07 & 5.66 & 3.01 & $\mathrm{Up}$ & 0.01451 & 0.03061 \\
\hline hsa-mir-205 & 12.86 & 10.57 & 4.89 & Down & 0.01510 & 0.03142 \\
\hline hsa-mir-34b & 4.01 & 4.99 & 1.98 & $\mathrm{Up}$ & 0.01815 & 0.03540 \\
\hline hsa-mir-486 & 5.60 & 4.49 & 2.15 & Down & 0.02010 & 0.03746 \\
\hline hsa-mir-320c-2 & 2.39 & 3.54 & 2.23 & Up & 0.02149 & 0.03930 \\
\hline hsa-mir-138-2 & 4.28 & 5.23 & 1.93 & Up & 0.02176 & 0.03941 \\
\hline hsa-mir-9-1 & 4.98 & 5.90 & 1.89 & $\mathrm{Up}$ & 0.02829 & 0.04723 \\
\hline hsa-mir-9-2 & 4.97 & 5.89 & 1.89 & Up & 0.02993 & 0.04892 \\
\hline
\end{tabular}

* log-transformed RPM

$1 \mathrm{P}$-value adjusted based on the Benjamini-Hochberg procedure for false discovery rate control

PNMNR = Patients with No Metastasis and No Recurrence; PR = Patients with Recurrence.

A risk score was calculated that combined the expression levels of each of the five microRNAs (See Methods). The combination of these miRNAs yielded an AUC of $95.3 \%$ (95\% C.I.: $84 \%-99 \%$ ) to predict prostate cancer metastasis (Figure 1) in the discovery sample. When examined individually, miR301a had the highest predictive ability with an AUC of $91.0 \%$ (95\% C.I.: $78 \%-99 \%)$.

\section{Cohort Validation}

Of the 515 patients who were included in the study (and who were not part of the Discovery Set), qPCR measurements of the miRNAs could not be obtained on 24 tumour samples leaving 491 (95.3\%) patients for final analysis. Of the 491 patients, 167 (34.0\%) developed biochemical recurrence, and 25 (5.1\%) developed metastasis, after a median follow-up of 8.7 years (interquartile range: $5.1-10.8$ years). The median age at the time of surgery was 62.8 years, the median PSA was $6.5 \mathrm{ng} / \mathrm{mL}$ at the time of diagnosis (IQR $4.3-9.4 \mathrm{ng} / \mathrm{mL}$ ), and the majority of patients $(61.2 \%, \mathrm{n}=300)$ had cancer confined within the pros- tate. The majority of patients had Gleason Score 7 tumours $(67.0 \%, n=329)$. Baseline demographic and tumor characteristics are described in Table 1. Stage, grade and PSA level at diagnosis were important prognostic factors for recurrence or metastasis (Table 3).

The median miRNA expression panel risk score was significantly higher among patients who developed recurrence compared to patients who did not ( 0.87 versus $0.53, p<0.0001)$. Similarly, the miRNA risk score was also higher among patients who developed metastasis compared to controls $(1.00$ vs. 0.61, $\mathrm{p}<0.0001)$. To evaluate the clinical significance of the miRNA panel score, we grouped patients into those with a low and high miRNA risk score, based on the median value of the miRNA score distribution. Patients with a low miRNA score had a significantly higher 15-year biochemical recurrence-free survival rate $(74.6 \%, 95 \%$ C.I.: $64.2 \%-82.4 \%)$, compared to patients with a high score $(27.1 \%, 95 \%$ C.I.: $17.3 \%-37.8 \%$, $\mathrm{p}<0.0001$ ) (Figure 2A). The 15-year metastasis-free survival was also significantly higher in the low 
miRNA score group (98.8\%, 95\% C.I.: 94.9\%-99.7\%), compared to the high score group $(77.5 \%, 95 \%$ C.I.: $63.9 \%-86.4 \%, \mathrm{p}<0.0001$ ) (Figure 2B). After adjusting for grade, stage and PSA level, patients with a high

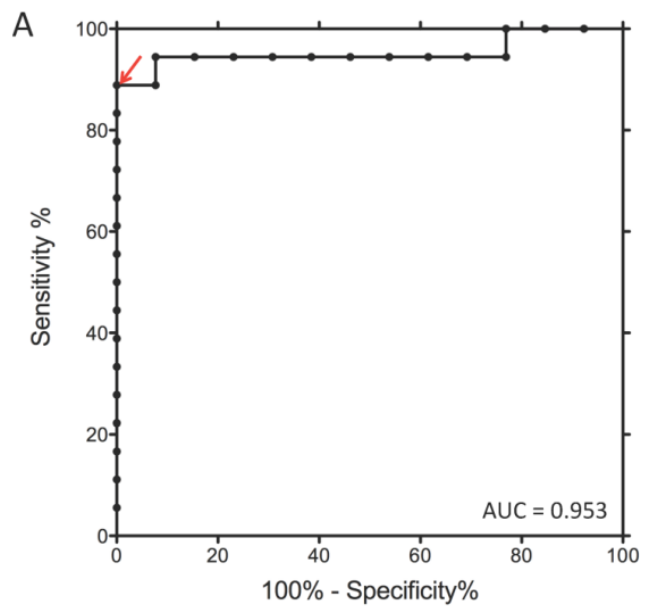

miRNA panel score had a higher rate of metastasis $(\mathrm{HR}=4.3$, 95\% C.I.: 1.7-11.1, $\mathrm{p}=0.002)$ and of biochemical recurrence $(\mathrm{HR}=2.6,95 \%$ C.I.: 1.8-3.6, $\mathrm{p}<0.0001)$ compared to those with a low score (Table 3 ).

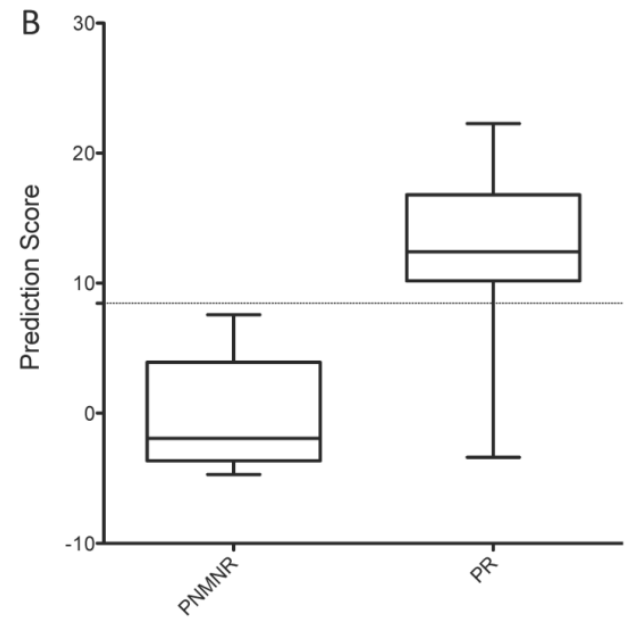

Figure 1: Receiver operating characteristic (ROC) curves for five-miRNAs scores in gene discovery set. A risk score for each patient was developed by linear combination of the expression level of each miRNA, weighted by the regression coefficients derived from the univariate logistic regression model based on the case-control design. The arrow indicates the selected score cutoff of 8.443 , which achieves AUC of 0.953 , the specificity of $100 \%$ and the sensitivity of $88.89 \%$. The boxplot demonstrates median and interquartile ranges of miRNA risk score for patients without (PNMNR) and with (PR) recurrence following radical prostatectomy. Note: PNMNR = Patients with No Metastasis and No Recurrence; PR = Patients with Recurrence.

Table 3: Univariate and multivariate Cox proportional hazard modeling of prognostic factors for prostate cancer recurrence and metastasis.

\begin{tabular}{|c|c|c|c|c|}
\hline Prognostic Factor & $\begin{array}{l}\text { Univariate Crude Hazard Ratio } \\
\text { ( } 95 \% \text { C.I.) }\end{array}$ & p-value & $\begin{array}{l}\text { Multivariate Adjusted Hazard } \\
\text { Ratio ( } 95 \% \text { C.I.) }\end{array}$ & p-value \\
\hline \multicolumn{5}{|l|}{ Biochemical Recurrence } \\
\hline \multicolumn{5}{|l|}{ MicroRNA Panel Score } \\
\hline Low & 1.0 & & 1.0 & \\
\hline High & $3.9(2.8-5.6)$ & $<0.0001$ & $2.6(1.8-3.6)$ & $<0.0001$ \\
\hline \multicolumn{5}{|l|}{ Histologic Grade (Gleason Score) } \\
\hline 6 & 1.0 & & 1.0 & \\
\hline 7 & $6.7(3.5-12.8)$ & $<0.0001$ & $3.4(1.7-6.7)$ & 0.0004 \\
\hline $8-10$ & $17.4(8.4-35.9)$ & $<0.0001$ & $4.9(2.2-10.9)$ & $(0.0001$ \\
\hline \multicolumn{5}{|l|}{ Pathologic Stage } \\
\hline Organ Confined (pT2) & 1.0 & & 1.0 & \\
\hline Extraprostatic Extension (pT3a) & $3.5(2.4-4.9)$ & $<0.0001$ & $2.2(1.5-3.1)$ & $<0.0001$ \\
\hline Seminal Vesicle Involvement (pT3b) & $8.2(5.4-12.3)$ & $<0.0001$ & $4.2(2.7-6.6)$ & $<0.0001$ \\
\hline \multicolumn{5}{|l|}{ PSA $(\mathrm{ng} / \mathrm{mL})$} \\
\hline$\leq 4$ & 1.0 & & 1.0 & \\
\hline $4-10$ & $2.5(1.5-4.0)$ & 0.0002 & $1.8(1.1-2.9)$ & 0.01 \\
\hline$>10$ & $3.0(1.8-5.0)$ & $<0.0001$ & $1.3(0.8-2.3)$ & 0.31 \\
\hline \multicolumn{5}{|l|}{ Metastasis } \\
\hline \multicolumn{5}{|l|}{ MicroRNA Panel Score } \\
\hline Low & 1.0 & & 1.0 & \\
\hline High & $13.4(3.1-56.8)$ & 0.0004 & $4.3(1.6-11.1)$ & 0.002 \\
\hline \multicolumn{5}{|l|}{ *Histologic Grade (Gleason Score) } \\
\hline $6-7$ & 1.0 & & 1.0 & \\
\hline $8-10$ & $16.4(7.4-36.2)$ & $<0.0001$ & $4.6(2.1-10.2)$ & 0.0002 \\
\hline \multicolumn{5}{|l|}{${ }^{*}$ Pathologic Stage } \\
\hline pT2 or $\mathrm{pT} 3 \mathrm{a}$ & 1.0 & & 1.0 & \\
\hline pT3b & $9.2(4.1-20.5)$ & $<0.0001$ & $2.8(1.4-5.3)$ & 0.003 \\
\hline \multicolumn{5}{|l|}{${ }^{*} \mathrm{PSA}(\mathrm{ng} / \mathrm{mL})$} \\
\hline$\leq 10$ & 1.0 & & 1.0 & \\
\hline$>10$ & $3.4(1.5-7.4)$ & 0.002 & $1.1(0.5-2.6)$ & 0.81 \\
\hline
\end{tabular}

* Groups collapsed from three categories into two due to sample size limitations among patients who developed metastasis. 
A

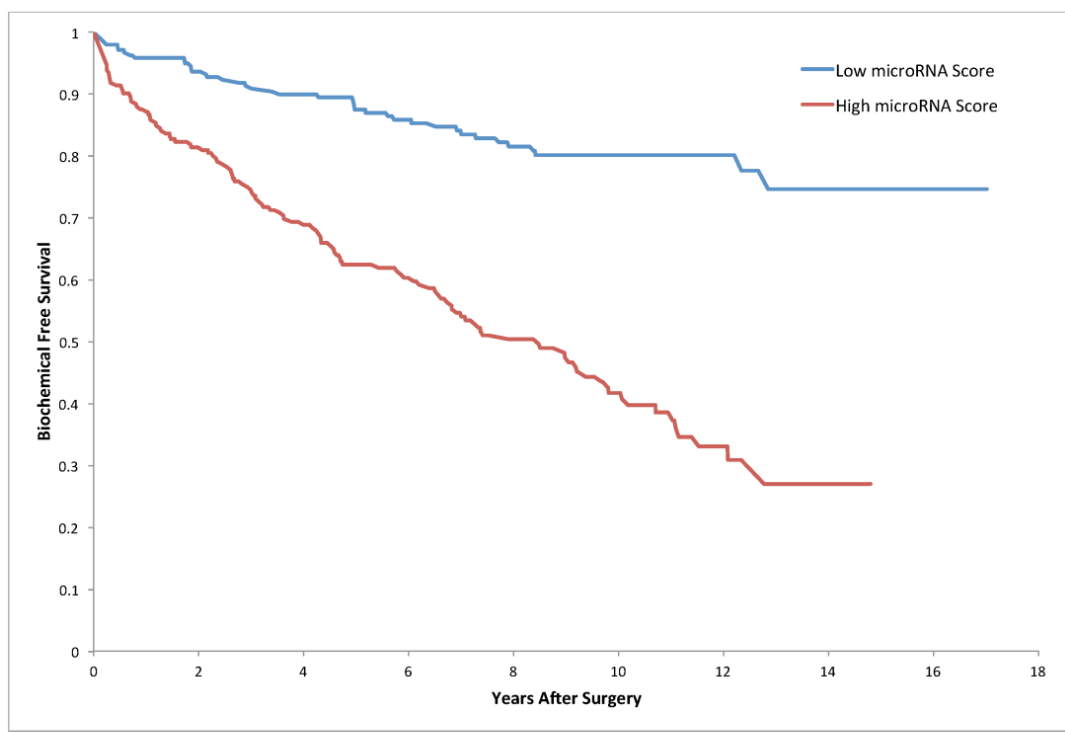

B

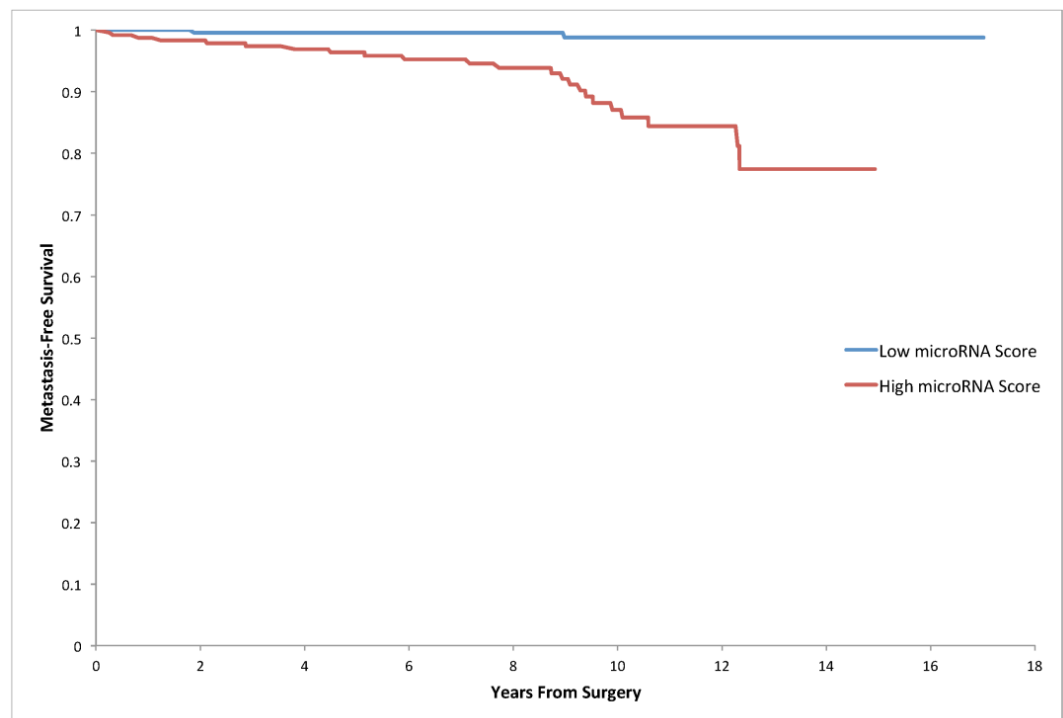

Figure 2: Kaplan-Meier survival analysis based on the five miRNA score panel. Prediction of biochemical recurrence free survival (A) and metastasis free survival (B) in all prostate cancer patients using five-miRNA panel, adjusted for clinical information.

We further examined the effect of the miRNA panel score in subgroups defined by stage and grade. In particular, we focused on a subgroup of patients with low-risk disease. Among patients with stage pT2 and negative surgical margins, who are at low risk of recurrence, the hazard ratio for a high miRNA panel score was 9.6 (95\% C.I. 3.6-25.3, p<0.0001) compared to patients with a low panel score (Table 4$)$. The absolute risk for recurrence based on high versus low miRNA panel score was $45.0 \%$ at 15 years. Among patients with low or moderate grade, patients with a high microRNA score experienced a high relative rate of recurrence (adjusted hazard ratio 9.4, 95\% C.I. 3.6-25.0, $\mathrm{p}<0.0001$ ), with an absolute risk increase of $33.1 \%$ (Table 4 ).

Among the five microRNAs, each microRNA was predictive of prostate cancer recurrence within this independent cohort. Among the five, miR-301a had the strongest association with prostate cancer recurrence from univariate Cox regression analysis (parameter estimate $=1.07$, chi-square $=34.2, p<0.0001$ ). Growth curves of PC3 cells transfected with each of the five miRNAs indicated that miR301a affects their proliferation (Figure 3). Because upregulated miRNAs are easier to detect and control therapeutically with antagomirs they are more clinically relevant candidates for preliminary and individual testing. Here we began with functional testing of miR301a in xenograft and in vitro prostate cancer models because, among the three upregulated miRNAs in the signature panel, miR301 had a significantly greater AUC than miR-454 and miR-652. 


\section{Mir-30 la promotes growth and metastasis of xenografted PC3 and LNCaP prostate cancer cells}

MiR-301a or empty vector control were stably overexpressed in PC3 and LNCaP prostate cancer cells. Expression of miR-301 in pools of transfected cells were 2 to 4 fold higher than in empty vector transfected cells, and up to six-fold higher in some clones (Figure 3). Overexpression of miR-301a affected in vivo tumor growth of xenografted PC3 and $\mathrm{LNCaP}$ cells. We found the average tumor volume was significantly greater (approximately 5-10-fold) in xenografts derived from miR-301a overexpressing cells compared to vector controls (Figure 3). Lung metastases were seen in host mice four weeks after the primary tumours were removed (Figure 4). Serial sections of host mouse lung tissue were stained for human-specific low molecular weight keratin or H\&E. Brown stain indicates the presence of metastatic human cells in host lung tissues. Quantitative imaging scores for anti-human-low molecular weight keratin in lungs of miR-301 transfected LNCaP and PC3 cells revealed that human cells are undetectable in lungs of control mice injected with empty vector transfected LNCaP and PC3 cells. Stained sections of lungs from mice bearing miR-301 transfected PC3 cells had four-fold more human cells/field than lung sections from mice injected with miR-301 transfected LNCaP cells (Figure 4E).

Table 4: Subgroup analysis of microRNA panel score by stage and grade.

\begin{tabular}{|c|c|c|}
\hline Risk Group Based on Adjuvant/Salvage Radiation Treatment Guidelines ${ }^{27}$ & $\begin{array}{l}\text { Adjusted Hazard Ratio based on MicroRNA score } \\
\text { High vs. Low ( } 95 \% \text { C.I.) }\end{array}$ & p-value \\
\hline pT2 with negative surgical margins $(n=169)$ & $9.6(3.6-25.3)$ & $<0.0001$ \\
\hline pT3a, pT3b or positive surgical margins $(n=274)$ & $2.3(1.5-3.3)$ & $<0.0001$ \\
\hline \multicolumn{3}{|l|}{ Risk Group Further Stratified by Histologic Grade } \\
\hline \multicolumn{3}{|l|}{ pT2 with negative surgical margins } \\
\hline Gleason Score 6-7 $(n=165)$ & $9.4(3.6-25.0)$ & $<0.0001$ \\
\hline Gleason Score 8-10 $(n=4)$ & -- & -- \\
\hline \multicolumn{3}{|l|}{ pT3a, pT3b or positive surgical margins } \\
\hline Gleason Score 6-7 $(n=232)$ & $2.0(1.4-3.1)$ & 0.0006 \\
\hline Gleason Score 8-10 $(n=42)$ & $3.4(1.0-11.6)$ & 0.05 \\
\hline
\end{tabular}
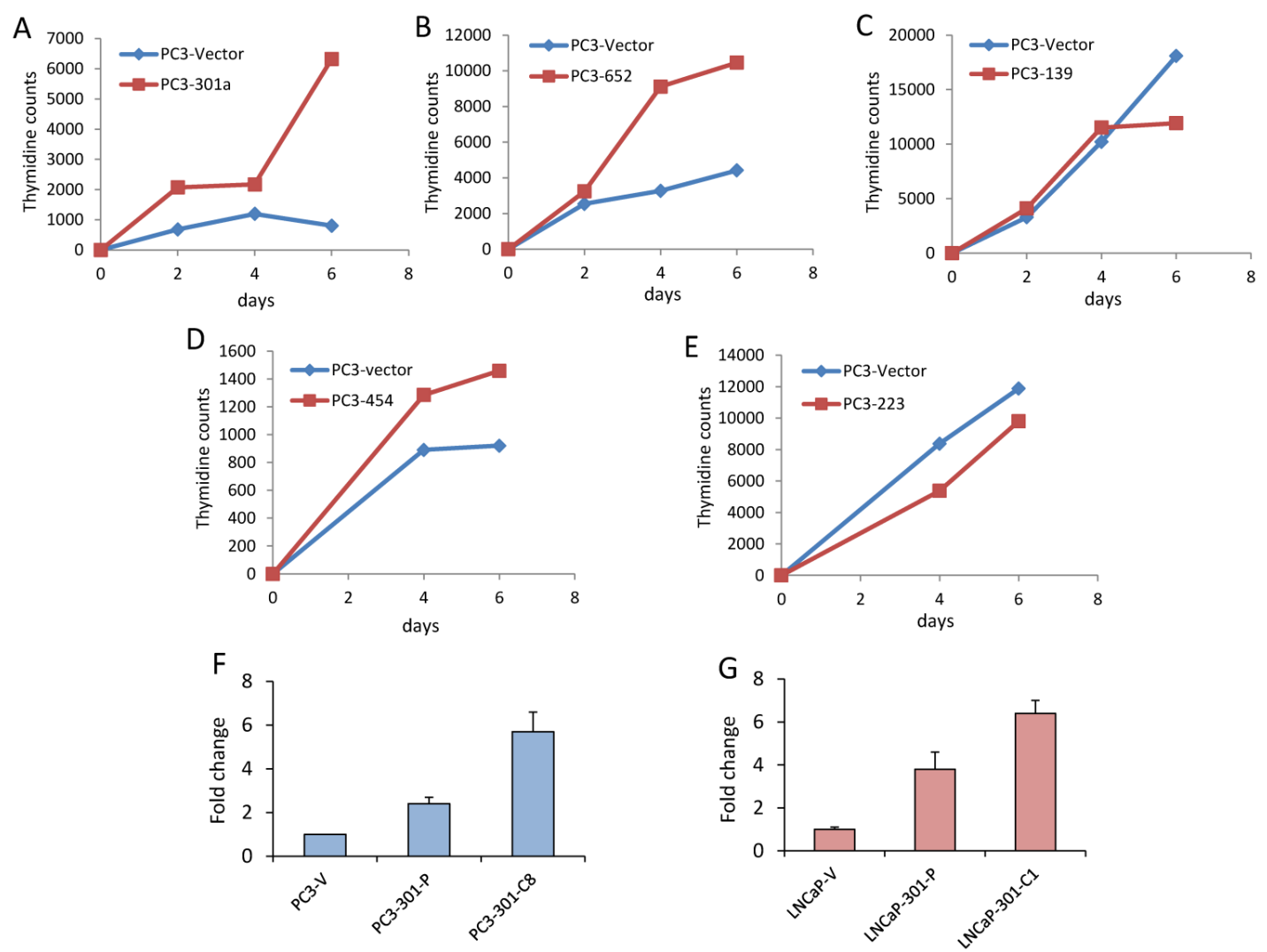

Figure 3: Growth curves of PC3 cells overexpressing each of the 5 miRNAs predictive of prostate cancer recurrence and metastasis. Graphical representation of tritiated thymidine incorporation by PC3 cells stably overexpressing (A) miR-301a, (B) miR-652, (C) miR-139, (D) miR-454, and (E) miR-223. Experiments were performed in triplicate. Expression of miR-30la in stably transfected pool and clones of PC3 (F) and LNCaP (G) cells. PC3 or LNCaP cell lines, as indicated, were stably transfected with miR-301a expression plasmid. Stably transfected cell lines were further subcloned from pools, resulting in the PC3-miR-301a subclone, C8, or the LNCaP-miR-30la subclone, $\mathrm{Cl}$. Levels of miR-30la specific expression were measured by quantitative PCR relative to endogenous control miR-28. 
A

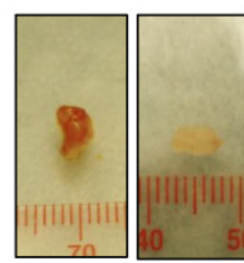

Vector Control

B

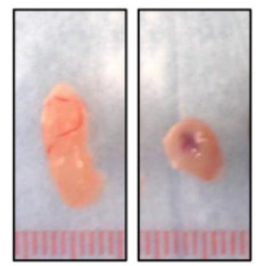

Vector Control

C

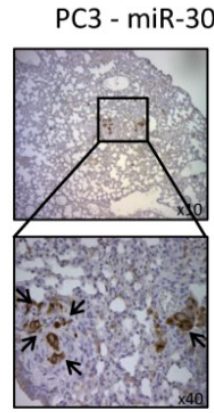

Anti Human LMW Cytokeratin
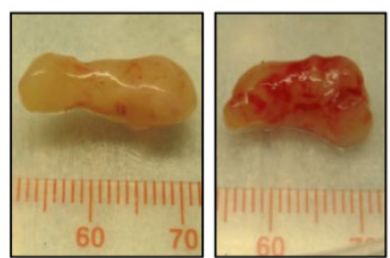

PC3 - miR-301a
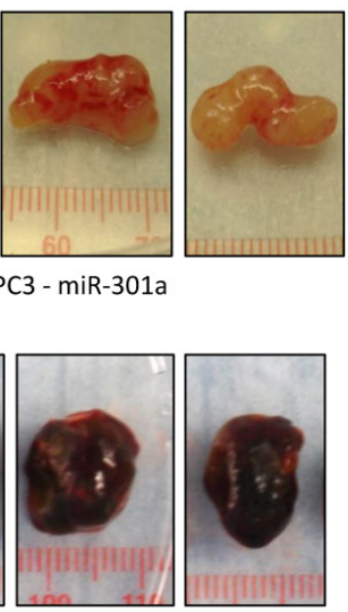

LNCaP - miR-301a

D

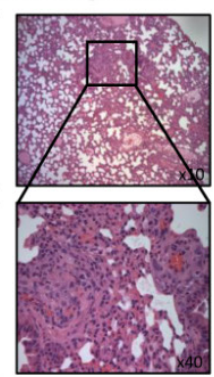

H.E. staining

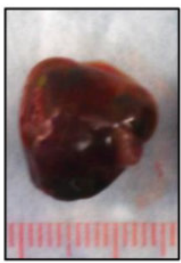

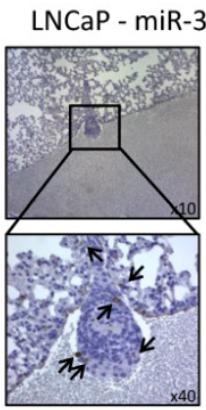

Anti Human LMW Cytokeratin

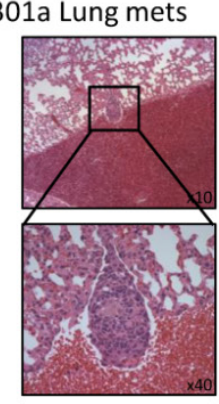

H.E. staining

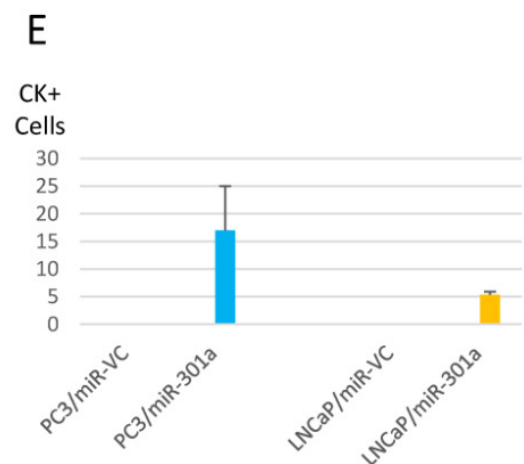

Figure 4: Overexpression of miR-301a increases tumorigenicity and metastasis of PC3 and LNCaP prostate cells. NOD/SCID mice were injected subcutaneously in the right flank with either stably transfected empty vector or miR-301 a expressing PC3 (1.0x106 cells/mouse) or LNCAP cells ( $2.5 \times 106$ cells/mouse) in $50 \%$ matrigel. At 4 weeks, some tumors were harvested and volumes were calculated. Representative tumors from PC3 (A) or LNCaP (B) injected mice are shown. (C) and (D) Representative lung tissue sections with metastasis stained with $\mathrm{H} \& \mathrm{E}$ or anti-human low molecular weight cytokeratin as indicated. (E). Quantitative imaging scores for anti-human-low molecular weight keratin in lungs of miR-301 transfected PC3 and LNCaP cells.

\section{Discussion}

In this study, we showed that among men treated with surgery for localized prostate cancer, microRNA expression from prostate cancer tissue is an important predictor of prostate cancer recurrence and metastasis, independent of grade, stage and PSA level at diagnosis. We defined a panel of five microRNAs from a genome wide search that can potentially be used to identify patients who have a high (or low) risk of developing prostate cancer recurrence and metastasis prior to initial treatment. Men with prostate cancer tumours that have a high panel miRNA score have up to a 4-fold risk of developing metastasis, compared to patients with tumours that have a low panel score. This miRNA panel can also identify patients at risk for recurrence among patients who would presumably be cured based on pathologic stage and grade.

In our analysis, Gleason score was the strongest independent predictor of recurrence and metastasis.
However, there is considerable evidence that patients with the same Gleason score may experience vastly different clinical outcomes $(17,18)$. The microRNA risk panel described here provides a significant additional prognostic factor after accounting for Gleason score, stage and PSA level at diagnosis and may significantly alter patient care if further validated. For patients who undergo surgery, this miRNA panel may be able to identify patients who will need additional treatment such as radiation therapy. Salvage or adjuvant radiation therapy have been shown to improve prostate cancer survival (19). In our cohort, patients with organ confined disease with negative margins who would not undergo radiation treatment based on current guidelines (19), had an increased risk for recurrence with a high miRNA panel score. Our findings could be used as an important prognostic indicator in this setting. In other cases, patients with clinically-localized prostate cancer are offered surveillance rather than surgery or radiation in order to avoid unnecessary treatment (20). The approach is not 
without risk, given that we have limited ability to distinguish between patients who have indolent or lethal forms of prostate cancer. Analyzing the microRNA profile of patients from the prostate biopsy specimen at the time of diagnosis could have the potential to identify patients at high risk for developing prostate cancer metastasis and who are not suitable for active surveillance. It will be important to validate this microRNA panel among an active surveillance cohort based on analyzing the needle biopsy tumour sample.

While this research was being undertaken, a number of other groups have examined the prognostic role of miRNAs in prostate cancer (5-8). The majority of these were conducted in small clinical cohorts and many assessed surrogate endpoints such as biochemical recurrence. To the best of our knowledge, this study represents the largest validation sample. Song et al conducted a similar expression profile analysis using next generation sequencing and found a panel of six miRNAs, none of which overlapped with our finding (21). This analysis was among ten patients between cases and controls with no validation cohort. Lichner et al examined a list of 754 miRNAs among 105 patients treated with surgery for prostate cancer (22). Three miRNAs that did not include those from our panel were identified to be associated with recurrence.. They used a discovery set comprising 41 patients and a validation cohort comprising 64 patients. However, they assessed early versus late biochemical recurrence as their outcome. As only a small percentage of patients with biochemical recurrence have systemic progression or die of their disease (23), this is likely not a clinically-relevant endpoint. Several studies have examined individual or a group of miRNAs, but are mainly based on in vitro discovery models. Currently, no putative miRNA has been used for clinical applications.

Deregulation of miRNAs has been shown to contribute to the development of many different human cancers, including breast, lung, liver, brain, gastric and colorectal cancer (24-29). In prostate cancer, some miRNAs act as oncogenes while others act as tumor suppressors. In this study we identified miR-301a as a novel oncogenic miR in aggressive prostate cancer, and the strongest out of the five-microRNA panel. MiR-301a has previously been shown to be associated with promotion of pancreatic, breast, gastric, colorectal, and hepatocellular carcinoma $(12,30-35)$. In prostate cancer, Xie et al. have shown that pre-adipocytes can increase prostate cancer metastasis through miR-301a modulation (36). Research in breast (37) and colorectal cancer (38) has shown that mir-301 may exert oncogenic effects through PTEN (37) and TGFBR2 pathways (38).
We found that miR-301a is upregulated in metastatic prostate cancer patient tumours compared to tumors from patients with no metastasis and no recurrence. Overexpression of miR-301a in PC3 and LNCaP prostate cancer cells was associated with increased growth rate. Further, xenografted prostate cancer cells overexpressing miR-301a resulted in much larger tumors than those derived from empty vector control cells, and metastatic lesions were found in lung tissue. A further investigation of all the effects of all five miRNAs individually and together in vitro and in vivo is desirable. Because, upregulated miRNAs are easier to detect and control therapeutically with antagomirs they are more clinically relevant candidates for treatment of patients. Here we began with functional testing of miR301a because, among the three upregulated miRNAs in the signature panel, miR301 had a significantly greater AUC than miR-454 and miR-652. Further study will be required to elucidate the biologic mechanism of miR-301a and the other four miRNAs in causing prostate cancer metastasis.

A limitation of microRNA profiling from human prostate cancer tumour tissue is the differential expression of microRNAs based on Gleason grading. From our study, we showed significant correlations between Gleason score and the microRNA panel score. Others have also shown differences in miRNA expression profiles by Gleason grade $(39,40)$. Other tumours within the prostate gland could have different miRNA expression patterns due to intra-tumour heterogeneity. Haffner et al recently characterized a lethal cell clone to originate from a small focus of low grade cancer rather than the primary tumour among one patient who died from prostate cancer (41). Further study will be necessary to elucidate how to identify a representative area of the prostate cancer tissue to measure the miRNA expression profile, as no standardized approach has been developed.

\section{Conclusions}

Based on a genome wide analysis and supported by validation in a large independent cohort, we have identified a novel panel of 5 miRNAs which are associated with prostate cancer prognosis. For men undergoing surgery for prostate cancer, this panel may identify those who are likely to benefit from additional therapy. Further, this discovery has enabled investigation into the underlying biology which distinguishes indolent and aggressive forms of this disease. With further research, such a panel may be able to distinguish patients who are suitable for active surveillance from those who are likely to progress without local therapy. 


\section{Acknowledgment}

This study was funded by the Edmond Odette Foundation, Cancer Research Society, the Ajmera Family Chair in Urologic Oncology and the Sunnybrook Health Sciences Centre Foundation.

\section{Authors' Contributions}

R.K.N., S.A.N. and A.S. composed the research question. R.K.N. and L.H.K. contributed patients who participated in the study. C.S., L.S. and M.N. performed histologic evaluation of the prostatectomy tissue and selected regions for extraction. J.S.B. performed data acquisition. Y.A. performed the genome wide sequencing. T.B., W.Y. and A.Z. performed the transfection of the cell lines and xenograft experiments. C.J.D.W., S.B. and R.K.N. performed data analysis. R.K.N., C.J.D.W. and A.S. drafted the manuscript and L.H.K. and S.A.N. critically revised the manuscript. All authors read and approved the final manuscript.

\section{Competing Interests}

The authors have declared that no competing interest exists.

\section{References}

1. Nam RK, Cheung P, Herschorn S, Saskin R, Su J, Klotz LH, et al. Incidence of complications other than urinary incontinence or erectile dysfunction after radical prostatectomy or radiotherapy for prostate cancer: a population-based cohort study. The lancet oncology. 2014;15(2):223-31.

2. D'Amico AV, Whittington R, Malkowicz SB, Schultz D, Blank K, Broderick $\mathrm{GA}$, et al. Biochemical outcome after radical prostatectomy, external beam radiation therapy, or interstitial radiation therapy for clinically localized prostate cancer. JAMA : the journal of the American Medical Association. 1998;280(11):969-74.

3. Lu J, Getz G, Miska EA, Alvarez-Saavedra E, Lamb J, Peck D, et al. MicroRNA expression profiles classify human cancers. Nature. 2005;435(7043):834-8.

4. Bartel DP. MicroRNAs: genomics, biogenesis, mechanism, and function. Cell. 2004;116(2):281-97.

5. Casanova-Salas I, Rubio-Briones J, Calatrava A, Mancarella C, Masia E, Casanova J, et al. Identification of miR-187 and miR-182 as biomarkers of early diagnosis and prognosis in patients with prostate cancer treated with radical prostatectomy. The Journal of urology. 2014;192(1):252-9.

6. Formosa A, Markert EK, Lena AM, Italiano D, Finazzi-Agro E, Levine AJ, et al. MicroRNAs, miR-154, miR-299-5p, miR-376a, miR-376c, miR-377, miR-381, miR-487b, miR-485-3p, miR-495 and miR-654-3p, mapped to the $14 q 32.31$ locus, regulate proliferation, apoptosis, migration and invasion in metastatic prostate cancer cells. Oncogene. 2014;33(44):5173-82.

7. Schubert M, Spahn M, Kneitz S, Scholz CJ, Joniau S, Stroebel P, et al. Distinct microRNA expression profile in prostate cancer patients with early clinical failure and the impact of let-7 as prognostic marker in high-risk prostate cancer. PloS one. 2013;8(6):e65064.

8. Spahn M, Kneitz S, Scholz CJ, Stenger N, Rudiger T, Strobel P, et al. Expression of microRNA-221 is progressively reduced in aggressive prostate cancer and metastasis and predicts clinical recurrence. International journal of cancer Journal international du cancer. 2010;127(2):394-403.

9. Nam RK, Zhang WW, Siminovitch KA, Kattan MW, Seth A, Klotz LH, et al. New Variants at 10q26 and 15q21 Are Associated With Aggressive Prostate Cancer in a Genome-Wide Association Study from a Prostate Biopsy Screening Cohort. Cancer, Biology and Therapy. 2011;12(11):997-1004.

10. Lossos IS, Czerwinski DK, Alizadeh AA, Wechser MA, Tibshirani R, Botstein $\mathrm{D}$, et al. Prediction of survival in diffuse large-B-cell lymphoma based on the expression of six genes. The New England journal of medicine. 2004;350(18):1828-37.

11. Yu SL, Chen HY, Chang GC, Chen CY, Chen HW, Singh S, et al. MicroRNA signature predicts survival and relapse in lung cancer. Cancer cell. 2008;13(1):48-57

12. Shi W, Gerster $\mathrm{K}$, Alajez NM, Tsang J, Waldron L, Pintilie $M$, et al. MicroRNA-301 mediates proliferation and invasion in human breast cancer. Cancer Res. 2011;71(8):2926-37.
13. Fan $\mathrm{Q}, \mathrm{He} \mathrm{M}$, Deng $\mathrm{X}, \mathrm{Wu}$ WK, Zhao L, Tang J, et al. Derepression of c-Fos caused by microRNA-139 down-regulation contributes to the metastasis of human hepatocellular carcinoma. Cell biochemistry and function. 2013;31(4):319-24.

14. Guo H, Hu X, Ge S, Qian G, Zhang J. Regulation of RAP1B by miR-139 suppresses human colorectal carcinoma cell proliferation. The international journal of biochemistry \& cell biology. 2012;44(9):1465-72.

15. Shen $K$, Liang $Q$, Xu K, Cui D, Jiang L, Yin P, et al. MiR-139 inhibits invasion and metastasis of colorectal cancer by targeting the type I insulin-like growth factor receptor. Biochemical pharmacology. 2012;84(3):320-30.

16. Wong CC, Wong CM, Tung EK, Au SL, Lee JM, Poon RT, et al. The microRNA miR-139 suppresses metastasis and progression of hepatocellular carcinoma by down-regulating Rho-kinase 2. Gastroenterology. 2011;140(1):322-31.

17. Siadat F, Sykes J, Zlotta AR, Aldaoud N, Egawa S, Pushkar D, et al. Not all gleason pattern 4 prostate cancers are created equal: A study of latent prostatic carcinomas in a cystoprostatectomy and autopsy series. The Prostate. 2015.

18. Jung JW, Lee JK, Hong SK, Byun SS, Lee SE. Stratification of patients with intermediate-risk prostate cancer. BJU international. 2015;115(6):907-12.

19. Thompson IM, Valicenti RK, Albertsen P, Davis BJ, Goldenberg SL, Hahn C, et al. Adjuvant and salvage radiotherapy after prostatectomy: AUA/ASTRO Guideline. The Journal of urology. 2013;190(2):441-9.

20. Greene KL, Albertsen PC, Babaian RJ, Carter HB, Gann PH, Han M, et al. Prostate specific antigen best practice statement: 2009 update. J Urol. 2013;189(1 Suppl):S2-S11.

21. Song C, Chen H, Wang T, Zhang W, Ru G, Lang J. Expression profile analysis of microRNAs in prostate cancer by next-generation sequencing. Prostate. 2015.

22. Lichner Z, Fendler A, Saleh C, Nasser AN, Boles D, Al-Haddad S, et al. MicroRNA signature helps distinguish early from late biochemical failure in prostate cancer. Clin Chem. 2013;59(11):1595-603.

23. Boorjian SA, Thompson RH, Tollefson MK, Rangel LJ, Bergstralh EJ, Blute ML, et al. Long-term risk of clinical progression after biochemical recurrence following radical prostatectomy: the impact of time from surgery to recurrence. European urology. 2011;59(6):893-9.

24. Bandres E, Cubedo E, Agirre X, Malumbres R, Zarate R, Ramirez N, et al. Identification by Real-time PCR of 13 mature microRNAs differentially expressed in colorectal cancer and non-tumoral tissues. Molecular cancer. 2006;5:29.

25. Ciafre SA, Galardi S, Mangiola A, Ferracin M, Liu CG, Sabatino G, et al. Extensive modulation of a set of microRNAs in primary glioblastoma. Biochem Biophys Res Commun. 2005;334(4):1351-8.

26. Guo J, Miao Y, Xiao B, Huan R, Jiang Z, Meng D, et al. Differential expression of microRNA species in human gastric cancer versus non-tumorous tissues. Journal of gastroenterology and hepatology. 2009;24(4):652-7.

27. Iorio MV, Ferracin M, Liu CG, Veronese A, Spizzo $R$, Sabbioni S, et al. MicroRNA gene expression deregulation in human breast cancer. Cancer Res. 2005;65(16):7065-70.

28. Murakami $\mathrm{Y}$, Yasuda $\mathrm{T}$, Saigo $\mathrm{K}$, Urashima $\mathrm{T}$, Toyoda $\mathrm{H}$, Okanoue $\mathrm{T}$, et al. Comprehensive analysis of microRNA expression patterns in hepatocellular carcinoma and non-tumorous tissues. Oncogene. 2006;25(17):2537-45.

29. Takamizawa J, Konishi H, Yanagisawa K, Tomida S, Osada H, Endoh H, et al. Reduced expression of the let-7 microRNAs in human lung cancers in association with shortened postoperative survival. Cancer Res. 2004;64(11):3753-6.

30. Chen Z, Chen LY, Dai HY, Wang P, Gao S, Wang K. miR-301a promotes pancreatic cancer cell proliferation by directly inhibiting Bim expression. J Cell Biochem. 2012;113(10):3229-35.

31. Liu L, Nie J, Chen L, Dong G, Du X, Wu X, et al. The oncogenic role of microRNA-130a/301a/454 in human colorectal cancer via targeting Smad4 expression. PloS one. 2013;8(2):e55532

32. Lu Z, Li Y, Takwi A, Li B, Zhang J, Conklin DJ, et al. miR-301a as an NF-kappaB activator in pancreatic cancer cells. The EMBO journal. 2011;30(1):57-67.

33. Wang M, Li C, Yu B, Su L, Li J, Ju J, et al. Overexpressed miR-301a promotes cell proliferation and invasion by targeting RUNX3 in gastric cancer. Journal of gastroenterology. 2013;48(9):1023-33.

34. Xu XD, He XJ, Tao HQ, Zhang W, Wang YY, Ye ZY, et al. Abnormal expression of miR-301a in gastric cancer associated with progression and poor prognosis. J Surg Oncol. 2013;108(3):197-202.

35. Zhou P, Jiang W, Wu L, Chang R, Wu K, Wang Z. miR-301a is a candidate oncogene that targets the homeobox gene Gax in human hepatocellular carcinoma. Digestive diseases and sciences. 2012;57(5):1171-80.

36. Xie H, Li L, Zhu G, Dang Q, Ma Z, He D, et al. Infiltrated pre-adipocytes increase prostate cancer metastasis via modulation of the miR-301a/androgen receptor (AR)/TGF-beta1/Smad/MMP9 signals. Oncotarget. 2015.

37. Ma F, Zhang J, Zhong L, Wang L, Liu Y, Wang $\mathrm{Y}$, et al. Upregulated microRNA-301a in breast cancer promotes tumor metastasis by targeting PTEN and activating Wnt/beta-catenin signaling. Gene. 2014;535(2):191-7.

38. Zhang $\mathrm{W}$, Zhang $\mathrm{T}$, Jin $\mathrm{R}$, Zhao $\mathrm{H}, \mathrm{Hu} J$, Feng $\mathrm{B}$, et al. MicroRNA-301a promotes migration and invasion by targeting TGFBR2 in human colorectal cancer. J Exp Clin Cancer Res. 2014;33(1):780.

39. Tsuchiyama $K$, Ito $H$, Taga $M$, Naganuma $S$, Oshinoya $Y$, Nagano $K$, et al. Expression of microRNAs associated with Gleason grading system in prostate cancer: miR-182-5p is a useful marker for high grade prostate cancer. Prostate. 2013;73(8):827-34 
40. Walter BA, Valera VA, Pinto PA, Merino MJ. Comprehensive microRNA Profiling of Prostate Cancer. Journal of Cancer. 2013;4(5):350-7.

41. Haffner MC, Mosbruger T, Esopi DM, Fedor H, Heaphy CM, Walker DA, et al.

Tracking the clonal origin of lethal prostate cancer. The Journal of clinical investigation. 2013;123(11):4918-22. 